\title{
Perioperative Upper Extremity Peripheral Nerve Injury and Patient Positioning: What Anesthesiologists Need to Know
}

\section{Kamel I* and Huck E \\ Lewis Katz School of Medicine at Temple University, USA}

*Corresponding author: Ihab Kamel, Lewis Katz School of Medicine at Temple University, MEHP 3401 N. Broad street, 3rd floor outpatient building ( Zone-B), Philadelphia, United States, Tel: 2158066599; Email: Ihab.kamel@tuhs.temple.edu

\section{Review Article}

Volume 4 Issue 3

Received Date: June 20, 2019

Published Date: August 01, 2019

DOI: $10.23880 /$ accmj-16000155

\section{Abstract}

Peripheral nerve injury is a rare but significant perioperative complication. Despite a variety of investigations that include observational, experimental, human cadaveric and animal studies, we have an incomplete understanding of the etiology of PPNI and the means to prevent it. In this article we reviewed current knowledge pertinent to perioperative upper extremity peripheral nerve injury and optimal intraoperative patient positioning.

Keywords: Nerve Fibers; Proprioception; Perineurium; Epineurium; Endoneurium; Neurapraxia; Ulnar Neuropathy

Abbreviations: PPNI: Perioperative Peripheral Nerve Injury; MAP: Mean Arterial Pressure; ASA CCP: American Society of Anesthesiology Closed Claims Project; SSEP: Somato Sensory Evoked Potentials

\section{Introduction}

Perioperative peripheral nerve injury (PPNI) is a rare complication with a reported incidence of $0.03-0.1 \%[1,2]$. PPNI is a significant source of patient disability and is the second most common cause of anesthesia malpractice claims $[3,4]$. Various types of studies have investigated PPNI including closed claims analyses, experimental animal models, observational studies, experimental volunteer studies and human cadaver studies. Despite these efforts, the mechanism of this injury is not completely understood [5]. In 2000, the ASA released a practice advisory for the prevention of peripheral nerve injury that was primarily based on expert consultant opinion [6]. The advisory was updated in 2011 and
2018.The most common perioperative peripheral nerve injuries involve the upper extremity with ulnar neuropathy and brachial plexus injury being the most frequent $[3,4]$. In this article we review upper extremity PPNI with regards to anatomy and physiology, mechanisms of injury, risk factors, and prevention of upper extremity PPNI.

\section{Anatomy and Physiology of Peripheral Nerves}

Peripheral nerves consist of bundles of nerve fibers (axons) that lie outside the pial covering of the central nervous system. These nerves include sensory afferent nerve fibers that connect receptors in the body to the central nervous system and motor efferent nerve fibers that connect the central nervous system to muscles or glands. Every nerve fiber is made up of many individual neurons, each with dendrites, cell bodies and axons. The sensory axons are either somatic or visceral and they 


\section{Anaesthesia \& Critical Care Medicine Journal}

innervate receptors in the skin, muscle, bone and viscera providing information in regard to touch, temperature, proprioception, pressure, and pain. When a nerve receives a stimulus to its dendrites from the CNS or a receptor in the body, an electrical impulse is created which moves down the dendrite, through the cell body and down the axon. At the tip of the axon, the neuron connects to other neurons by means of a chemical synapse, and the impulse is transmitted chemically to the next nerve(s).

Peripheral nerves are made up of multiple axons. Each axon is has a connective tissue sheath called the endoneurium. The axons are bundled into fascicles by a surrounding sheath called the perineurium. Finally, each peripheral nerve is made up of many fascicles enclosed in a dense connective tissue sheath called the epineurium. These connective tissue sheaths support the nerve structure and supply the axons with blood (vasonervorum) and lymph vessels. Schematic presentation of the cross-section of a peripheral nerve is depicted in (Figure 1). The larger nerves are more often myelinated while the smaller diameter nerves are unmyelinated. A myelinated nerve is surrounded by the plasma membrane of Schwann cells. This plasma membrane spirals around the axon in concentric layers. Each myelinated axon is surrounded by a series of Schwann cells along its length. Schwann cells meet at the Nodes of Ranvier. Generally, Schwann cells support axons by providing metabolites and tropic factors. Myelinated nerve fibers are also known as A fibers and they conduct signals much faster than unmyelinated nerve fibers due to the configuration of Schwann cells. Unmyelinated nerve fibers are named $\mathrm{C}$ fibers, are more common than A fibers and have a smaller diameter.

The blood supply to peripheral nerves is provided by the vasonervorum. These vessels are derived from branches of nerve-associated small arteries that form a plexus of vessels after they enter the epineurium. This plexus also penetrates the perineurium and the endoneurium as arterioles and capillaries. In experimental animal models, the blood supply to peripheral nerves has been shown to have no intrinsic autoregulation, thus blood flow to peripheral nerves is dependent on mean arterial blood pressure [7-9]. In experimental animal models, High blood flow in the sciatic nerve was observed between mean blood pressures of $80-110 \mathrm{mmHg}$ and marked decrease in the peripheral nerve blood flow was observed at mean blood pressures below $85 \mathrm{mmHg}$ [7]. However, normal blood flow exceeds the metabolic requirements of peripheral nerves by a favorable margin [10]. Thus, a significant reduction in the blood flow to the nerve is required to affect the conduction of impulse in the nerve. Acute nerve ischemia leads to impairment of impulse conduction across the nerve [11].

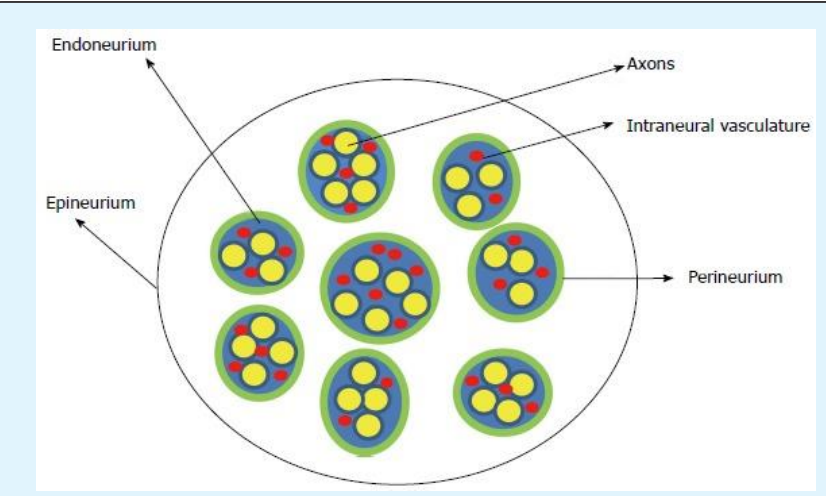

Figure 1: Schematic representation of the cross section of the peripheral nerve. Peripheral nerves are made up of multiple axons. The endoneurium is a connective tissue sheath surrounding the axons. The axons are bundled into fascicles and these have a surrounding sheath called the perineurium. Finally, each nerve is made up of many fascicles and is enclosed in a dense connective tissue sheath called the epineurium. These connective tissue sheaths support the nerve with associated blood vessels (vasonervorum).

\section{Mechanisms of Perioperative Nerve Injury}

Injury to a peripheral nerve can occur from damage to any or all of its parts, the nerve itself, the surrounding connective tissue, the Schwann cells, or the blood vessels supplying the peripheral nerve. Such injury can occur by compression or stretch of the nerve, or by loss of blood flow to the nerve resulting in ischemia. The etiology of PPNI is multifactorial and complex. Direct trauma to the peripheral nerve leading to disruption of nerve fibers will cause injury. However, with PPNI the most common etiology is believed to be ischemia of the peripheral nerve [12-24]. Stretch and compression of the peripheral nerve can cause ischemia [25]. During surgery patients are placed in positions that would not be tolerated in the nonanesthetized state. Such positions can stretch peripheral nerves of the upper extremity. Stretch of the peripheral nerve beyond $5-15 \%$ of its original length may lead to ischemia in the nerve and results in slowing of conduction in the peripheral nerve [26-30]. Stretch of the peripheral nerve leads to an increase in the intraneural pressure and 


\section{Anaesthesia \& Critical Care Medicine Journal}

compression of intraneural capillaries resulting in ischemia [28-33].

Direct external compressive forces may increase local tissue pressure leading to a reduction in perfusion pressure and subsequent ischemia. Depending on position, compression may also directly affect the local blood supply to the peripheral nerve. Reduction in the mean arterial pressure (MAP) can affect peripheral nerve function under general anesthesia and may decrease conduction in the peripheral nerve especially in the presence of compression or stretch $[34,35]$. Simultaneous compression at the point of contact with prominent bony structures and stretch may occur in certain positions. Magnetic resonance imaging in human cadavers revealed that the ulnar nerve is stretched over the medial epicondyle with incremental flexion of the elbow [36]. This stretch results in elongation of the ulnar nerve up to $18 \%$ greater than resting nerve length [36]. Perioperative ulnar neuropathy may be due to inflammation. A recent study by Staff et al suggests inflammation as an etiology for perioperative peripheral nerve injury. In this study, some patients with perioperative ulnar neuropathy had evidence of diffuse generalized microneuritis that improved significantly when treated with high-dose steroids [37].

Seddon has classified nerve injury as belonging to one of three types: neurapraxia, axonotmesis and neurotmesis. Neurapraxia is a mild degree of nerve damage that causes a failure of signal conduction through the injured segment of nerve. This type of injury is reversible. Axonotmesis involves a disruption of the axon of the nerve, but its supportive tissues including the endoneurium remain intact. This injury is also potentially reversible but requires neural regeneration. Finally, neurotmesis is the complete severing of the nerve: the axon and all its supporting connective tissues. This type of nerve injury has a poor prognosis for recovery [38].

\section{Risk Factors for Perioperative Peripheral Nerve Injury}

The etiology of PPNI is multifactorial. PPNI can occur due to predisposing factors such as patient co-morbidities and anatomical differences in association with precipitating factors that lead to direct damage to the nerve or more likely acute ischemia (Table 1). Male gender, extreme body habitus, prolonged hospitalization, diabetes mellitus, hypertension, tobacco use, general anesthesia, prone position, neurosurgical procedures and orthopedic procedures have all been associated with PPNI
[1,314]. Hypotension, hypoxia, dehydration, electrolyte disturbance and hypothermia have also been associated with PPNI [38].

\begin{tabular}{|c|}
\hline $\begin{array}{c}\text { Risk Factors associated with Perioperative } \\
\text { Peripheral Nerve Injury }\end{array}$ \\
\hline Male patients \\
\hline Prolonged hospital stay $>14$ days \\
\hline Extremes body habitus (BMI $<25$ or $>38$ ) \\
\hline Diabetes mellitus \\
\hline Chronic hypertension \\
\hline Tobacco use \\
\hline General anesthesia \\
\hline The prone position \\
\hline Neurosurgical procedures \\
\hline Orthopedic procedures \\
\hline Hypotension \\
\hline Hypoxia \\
\hline Dehydration \\
\hline Electrolyte disturbance \\
\hline Hypothermia \\
\hline
\end{tabular}

Table 1: Risk factors associated with perioperative peripheral nerve injury.

\section{The Closed Claims Analysis Project}

An important source of information on the occurrence and mechanisms of peripheral nerve injury has been the American Society of Anesthesiology Closed Claims Project (ASA CCP) $[3,4]$. This project was established in 1984 by the American Society of Anesthesiologists to identify anesthesia-related complications and their mechanisms in order to improve patient safety. The project was one response to rising professional liability insurance premiums; $11 \%$ of dollars paid for patient injury claims were related to anesthesiologists, while they represented only $3 \%$ of physicians in the United States [39]. The ASA CCP is a collection of evaluations of anesthesia-related injuries from the closed claims files of 35 professional liability insurance companies in the United States. These companies insure approximately $60 \%$ of practicing anesthesiologists in the United States. Each closed claim file is evaluated by at least one trained anesthesiologist who follows strict data collection instructions. Inclusion of a claim in the project depends on the availability of enough information to reconstruct the events leading to the claim to determine the nature and possibly the cause of the injury as well as the appropriateness of care.

These files typically contain hospital and medical records, narrative statements from healthcare personnel 


\section{Anaesthesia \& Critical Care Medicine Journal}

and expert witnesses, deposition summaries and the amount of the settlement or jury award. The data collection forms, summaries and determinations of appropriateness of care are then sent to two or three practicing anesthesiologists on the ASA CCP Committee for review. As of the year 2000, there were 5,480 claims in the ASA CCP (from 1961-99), dental injuries excluded [39]. Data from the ASA CCP can provide insight into the types and mechanisms of injury, but it also has limitations. Only claims in which information is deemed adequate can be added to the ASA CCP. Other limitations are the retrospective nature of the analysis, bias toward more severe injury (those injuries most likely to result in a malpractice action), and the representation of only $60 \%$ of practicing anesthesiologists. Also, the incidence of peripheral nerve damage cannot be determined from the information provided in the ASA CCP because it lacks a denominator of total surgical procedures (population at risk).

There have been two analyses of the ASA CCP to look at peripheral nerve damage in anesthesia-related injury. The first was published in 1990 and examined 1,541 files accumulated in the ASA CCP through May 1989. Of these files, 227 or $15 \%$ were related to nerve injury. Some nerves were more frequently injured than others. Injury to the ulnar nerve made up one third of total claims, while the brachial plexus (23\%) and lumbosacral nerve roots (16\%) were second and third most common injuries respectively [3]. Ten years later, another analysis was completed on 4,183 files in the ASA CCP. In this publication anesthesia-related nerve injury made up 16\% (670 claims) of the total number of claims, an insignificant change from previous findings [4]. The authors of the second analysis hypothesized that the reason the incidence of nerve injury has not changed over a decade is primarily due to the incomplete understanding of the mechanisms of nerve injury. Thus, consistent methods for intervention and prevention of injury are not feasible. Only a few claims expressly described the mechanism of injury. For example, 10 of the 113 ulnar nerve injury claims described the mechanism of injury. In $66 \%$ of all the files involving nerve injury, care was deemed appropriate despite the injury. In contrast, in only $42 \%$ of all other types of claims was care found to be appropriate [4].

An updated ASA CCP report of PPNI under general anesthesia (GA) between 1990 and 2013 showed that PPNI claims accounted for $12 \%$ (420 claims) of all anesthesia malpractice claims (3454 claims) [40]. Compared to other anesthesia claims, claims for PNI involved patients who are males, adults, healthier (ASA physical status I-II), and having elective procedures. Thirty-nine percent of PPNI claims associated with GA involved spine, nonspine orthopedic, cardiac and neurosurgery procedures. Eighty-four percent of PPNI associated with GA involved the upper extremity $(36 \%$ brachial plexus, $30 \%$ ulnar injury, $10 \%$ median, and $8 \%$ radial nerve) [40]. The mechanism of injury could not be identified in $6 \%$ of nerve injury claims while $39 \%$ of claims did have an identifiable cause [40].

\section{The incidence of Perioperative Peripheral Nerve Injury (PPNI)}

The incidence of PPNI cannot be determined from the ASA CCP; however, there have been a number of studies that have provided an estimate of the incidence of this injury. A retrospective analysis of 380,680 anesthetic cases at a tertiary care university hospital identified only 112 cases of peripheral nerve injury over a 10-year period, a rate of $0.03 \%$ [1]. In this study, PPNI was defined as a new sensory and/or motor deficit occurring within 48 hours of sedation or anesthesia. Three databases were examined to identify these injuries, the Department of Anesthesiology's closed claims files and Quality Assurance database, as well as the institution's outpatient and inpatient medical diagnoses and billing codes database. This low incidence of perioperative nerve injury may be an underestimation due to the definition of nerve injury used in the study. One prospective study looking at only ulnar nerve injury showed that this perioperative injury often does not manifest until more than 48 hours after surgery [41], so patients with as yet undiagnosed nerve injury may have been excluded in the retrospective study. Other studies have shown that injury to peripheral nerves during cardiac surgery may be much higher at 1.5\%-24\% for brachial plexus injury [42].

\section{Ulnar Neuropathy}

Despite the seeming lack of improvement in patient safety, much has been discerned in regard to peripheral nerve injury from the ASA CCP. According to the ASA CCP published in 1999, 28\% of PPNI malpractice claims involved the ulnar nerve suggesting that this nerve is the most commonly injured during the perioperative period. ${ }^{4}$ However, a recent update of the ASA CCP published in 2018, showed ulnar nerve injury as the second most common cause of PPNI related malpractice claims, responsible for $30 \%$ of all such claims in patients who had general anesthesia [40]. Signs and symptoms of an ulnar nerve injury include pain, paresthesias and/or weakness 


\section{Anaesthesia \& Critical Care Medicine Journal}

of the fourth and fifth digits; manifested as reduced ability to abduct these fingers or oppose the fifth digit. Patients may first complain of a clumsy hand with loss of sensation in the palm. Over time, the hypothenar eminence may atrophy. While the mechanism of injury to the ulnar nerve (and other peripheral nerves) was historically assumed to be due to malpositioning of the patient during the perioperative period, several studies have shown that the cause of this patient injury is multifactorial.

In 1987, a prospective study was published that found 17 of 6538 orthopedic surgery patients developed ulnar neuropathy postoperatively (all patients were followed for at least six months) [43]. All 17 patients had abnormal nerve conduction times in the contralateral ulnar nerve, suggesting that these patients may have had subclinical nerve dysfunction prior to surgery [43]. A retrospective study of 1,129,692 patients who underwent noncardiac surgical procedures over a 35-year period showed an incidence of ulnar neuropathy of 1 in 2729 patients or $0.037 \%$ [14]. Ulnar neuropathy in this study was defined as persisting for at least three months, so the calculated incidence did not include minor neuropathy symptoms that resolved quickly. This same study identified patient risk factors not previously reported. Patients with ulnar nerve injury were found to be predominantly male (70\%), thin or obese $(\mathrm{BMI}<25$ or $>38$ ), and had a length of hospitalization of more than 14 days. In this study most neuropathies were diagnosed more than 24 hours after surgery.

Of the 380 patients who were followed after the initial study, 53\% had complete resolution of their symptoms within one year of surgery. Six percent regained complete sensory and motor recovery but had residual paresthesia. Patients with sensory deficits had an $80 \%$ incidence of recovery compared to a recovery incidence of $35 \%$ for those with mixed motor and sensory [14]. In addition, a prospective study of ulnar neuropathy in 1,502 surgical patients confirmed male predominance and prolonged hospitalization as risk factors for developing ulnar neuropathy [41]. In this study, the incidence of neuropathy was $0.5 \%$ (7 patients). All 7 patients developed symptoms more than 48 hours after surgery. Of the seven patients identified in this study, 4 had resolution of their symptoms within 6 weeks (57\%), while the remaining 3 had persistent symptoms 2 years later [41].

The risk factor of prolonged hospitalization was further investigated by a prospective study looking at ulnar nerve injury in medical patients. This prospective study looked at 990 medical patients admitted to a university hospital system over a three-month period who agreed to participate in the study [44]. All patients were free of ulnar neuropathy symptoms. Patients were assessed daily during the hospital stay and by questionnaire after discharge. Of the 990 patients, 3 were identified for further evaluation by a neurologist, and 2 were diagnosed with ulnar neuropathy (incidence of $0.2 \%$ ). Both of these patients were male, over the age of 50 , had symptoms arise 3-4 days after admission and selfreported lying primarily in a supine position during hospitalization [44]. Clearly, perioperative ulnar neuropathy has a multifactorial cause, with patient anatomy and time spent in the supine position (measured in days) being significant risk factors. The prognosis for recovery from such an injury is approximately 50\%. The higher incidence of perioperative ulnar neuropathy in males can be explained by anatomical differences. Men have a thickened flexor retinaculum, significantly larger coronoid tubercle and significantly less fat content in the medial aspect of the elbow compared to women $[45,46]$. Men are more susceptible to direct pressure on the ulnar nerve fibers than women [47]. Swenson et al investigated the relative sensitivity of ulnar, median and radial nerve to ischemia using somatosensory evoked potentials (SSEP) monitoring under general anesthesia in the supine position. The investigators concluded that compared to the median and radial nerves, the ulnar nerve were relatively more sensitive to ischemia [22].

\section{Brachial Plexus Injury}

According to the ASA CCP published in 1999, the brachial plexus was the second most common peripheral nerve injury reported making up 20\% of all nerve injury claims [4]. A recent update of the ASA CCP now shows brachial plexus injury as the most common cause of malpractice claims, responsible for $36 \%$ of all nerve injury malpractice claims in patients who had general anesthesia [40]. Symptoms of a brachial plexus injury include pain, parasthesias and weakness of shoulder and upper arm muscles as well as similar symptoms of the wrist and fingers. The incidence of brachial plexus injury in non-cardiac surgery is reported to be $0.02 \%$ [48]. Positioning is thought to play an important role in brachial plexus injury. The plexus has a superficial location and passes through two fixed points of anatomy, the intervertebral foramen and the axillary sheath, which place it at risk for stretch and compression during surgery. Stretch can occur during median sternotomy, when the arm is abducted to $>90^{\circ}$, or if the head is turned away from the plexus at risk. Compression of the nerves 


\section{Anaesthesia \& Critical Care Medicine Journal}

between the clavicle and the first rib can also occur due to shoulder braces [49]. Lateral tilt of the head and excessive abduction on the non-dependent arm in the lateral decubitus position may stretch the brachial plexus leading to injury [50]. Prognosis for recovery from a perioperative brachial plexus injury is unknown, though it may be similar to that for ulnar nerve injury.

\section{Median Neuropathy}

According to the ASA CCP reports, median nerve injury comprises 4-10\% of anesthesia-related malpractice cases for PPNI $[4,40]$. The median nerve passes through the carpal tunnel, and compression of the nerve can occur in this area with even minor swelling. In addition, hyperextension of the wrist may cause both stretch and compression of the nerve. However, stretch is the main mechanism of perioperative median nerve injury. Median neuropathy commonly occurs in men between the ages of 20-40 years of age [46]. Men with large and welldeveloped biceps or with reduced range of elbow extension may be at greater risk when positioned in certain positions under anesthesia. They may have reduced flexibility and an inability to fully extend the elbow. This results in shortening of the median nerve over time. During general anesthesia, when the biceps is relaxed, the elbow may be stretched to an extent that would not be tolerated by the patient in the awake state. This stretching action may lead to median nerve injury [46]. Symptoms of median nerve injury consist of pain or sensory and/or motor deficits of the thumb and the first two and a half fingers as well as the palm of the hand. The ability to oppose the thumb and fifth digit is usually compromised. Median neuropathies do not have a favorable prognosis compared to the ulnar and radial nerve. Motor dysfunction was sustained up 2 years after the initial onset of symptoms in $80 \%$ of patients with median neuropathies. Nerve conduction studies on the median nerve when the wrist is hyperextended (simulating the position for arterial line placement) revealed that $83 \%$ of subjects had a decrease in sensory action potential amplitude after approximately 40 minutes [51].

\section{Radial Neuropathy}

Radial nerve injury accounts for 3-8\% of nerve injury identified in the ASA CCP $[4,40]$. Compression of the radial nerve in the mid-humerus region (spiral groove) is considered the main mechanism of injury [46]. This may occur due to compression of the radial nerve by vertical bars of abdominal retractors in the supine arms out position. In the lateral decubitus position, the vertical bars supporting the overhead arm board may compress the dependent radial nerve at the radial groove while the non-dependent radial nerve may be compressed by the overhead arm board if it protrudes into the mid-humerus. The radial nerve may be compressed and injured when the arm loses support, slips and the weight of the upper extremity is supported against the edge of the arm board or operating room table by the mid-humerus. Injury of the radial nerve may result in wrist drop or paresthesias in the dorsal side of the hand. Compared to median neuropathies, radial neuropathy has a more favorable prognosis. About $50 \%$ of patients improve within 6 months, and in $70 \%$ of the patients, symptoms completely resolved within 2 years.

\section{Intraoperative Patient Positioning and Upper Extremity Nerve Function}

During surgical procedures patients are positioned for optimal operative-site exposure. The effect of operative positioning on upper extremity nerve function has been evaluated. Kamel IR, et al. used intraoperative SSEP to determine the relationship between 5 operative positions and the incidence of impending upper extremity peripheral nerve injury [34]. This retrospective study included 1000 consecutive spine surgeries in which SSEP monitoring was used. The investigators defined impending peripheral nerve injury as significant SSEP changes that returned to baseline after modification of upper extremity position. The authors concluded that $92 \%$ of significant SSEP changes could be reversed with an intervention by the anesthesiologist. Postoperatively none of the patients developed postoperative clinical perioperative peripheral nerve injury. Interventions employed by the anesthesiologist, once intraoperative impending nerve injury was identified, included position modification strategies to decrease shoulder abduction, correction of extreme elbow flexion and extension, release of shoulder traction on tucked arms and return of arms to their original position if the position had been modified prior to the SSEP change. The lateral decubitus position and the prone "superman" position were significantly associated with impending upper extremity peripheral nerve injury compared to the prone arms tucked, supine arms tucked and supine arms out positions (Figure 2). The incidence of position related impending upper extremity peripheral nerve injury in the prone superman (prone surrender) and the lateral decubitus position were $7 \%$ and $7.5 \%$ respectively. The incidence of position related impending perioperative peripheral nerve injury in the supine arms out and supine arms 


\section{Anaesthesia \& Critical Care Medicine Journal}

tucked were $3.2 \%$ and $1.8 \%$ respectively. The incidence of position related impending perioperative peripheral nerve injury in the prone arms tucked position was $2.1 \%$ [34].

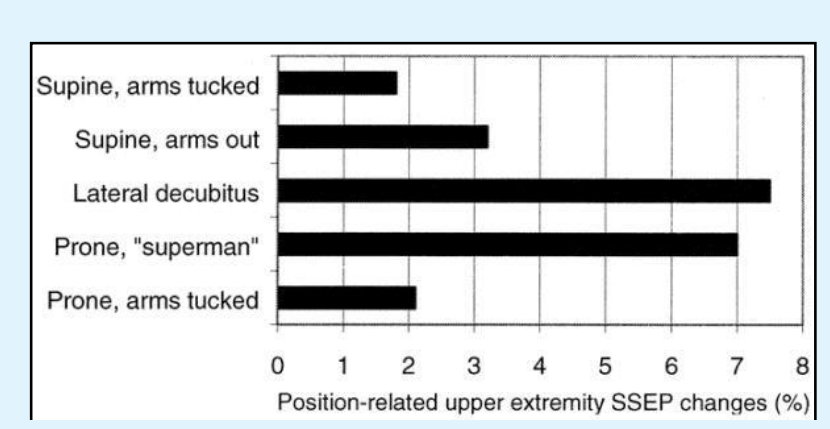

Figure 2: The incidence of intraoperative impending upper extremity nerve injury in 5 standard operative positions during spine surgery. The lateral decubitus position and the prone "superman" position were significantly associated with higher incidence of impending upper extremity peripheral nerve injury compared to the prone arms tucked, supine arms tucked and supine arms out positions.

\section{Upper Extremity Position and Peripheral Nerve Function}

The ulnar nerve traverses a superficial path at the medial epicondyle of the humerus and thus is vulnerable to direct external compressive forces. Prielipp et al investigated the influence of arm position on the degree of external pressure applied to the ulnar nerve at the elbow using a computerized pressure sensing mat, clinical paresthesias and SSEP monitoring in healthy awake volunteers. The investigators compared 3 arm positions (supination, neutral orientation and pronation). The same test was applied to an additional group of volunteers whose shoulder were placed in $30^{\circ}, 60^{\circ}$ and $90^{\circ}$ abduction. The authors concluded that forearm supination significantly minimized pressure over the ulnar nerve at the elbow (2 $\mathrm{mmHg}$ ) compared with neutral forearm orientation $(69 \mathrm{mmHg}$ ) and pronated forearm position $(95 \mathrm{mmHg})$. Neutral forearm orientation resulted in significantly less pressure over the ulnar nerve at the elbow compared to the pronated forearm position. In the neutral forearm orientation, pressure over the ulnar nerve decreased significantly when the shoulder was abducted from $0^{\circ}$ to $90^{\circ}$. In the supinated forearm position, direct pressure over the ulnar was low regardless of the degree of shoulder abduction. However, in the pronated forearm position, direct pressure over the ulnar nerve was high regardless of the degree shoulder abduction [52].

To support the empirical positioning recommendations designed to protect the brachial plexus, Coppieters et al investigated the effect of various arm and neck positions on the upper extremity peripheral nerves using the brachial plexus tension test (BPTT) in healthy awake male volunteers [53]. This experiment was designed to test the compliance of the brachial plexus and involved body positioning similar to that required for some intraoperative surgical positions. The range of elbow extension after arm abduction, wrist extension, and lateral rotation of the arm, cervical contralateral flexion or a combination of these moves was measured. The effect of fixation of the shoulder girdle with the above positions was also investigated. Pain or full extensions were the endpoints. Application of various arm and neck positions led to substantial discomfort during elbow extension and limitation of the extension range. The study showed that elbow extension decreased as more test components were added (multiple positioning moves). In particular, wrist extension and cervical contralateral lateral flexion significantly reduced the range of motion for elbow extension. Shoulder fixation resulted in a significantly reduced range of motion in all tests. The authors concluded that cervical contralateral flexion, depression of the shoulder girdle and lateral rotation of the shoulder, in combination with shoulder abduction and wrist extension, stresses the brachial plexus and had a significant effect on the range of elbow extension. The authors further concluded that simultaneous application of these variables may easily load the peripheral nervous system and compromise vital processes in the peripheral nerve. The finding of this study supported current positioning recommendations to protect the brachial plexus. Based on the finding of their study, the authors advocate that shoulder abduction and lateral rotation be kept to a minimum, the head should remain in the midline position, the arm should be kept at the side if possible, the elbow should be gently flexed and the forearm supinated. Wrist extension and shoulder braces should be avoided whenever possible [53].

\section{Upper Extremity Position and the Morphology of the Ulnar Nerve}

The ulnar nerve is the only peripheral nerve that passes on the extensor side of the joint. This may lead to stretch of the ulnar nerve with progressive elbow flexion. Elbow flexion may lead to a significant decrease in the 


\section{Anaesthesia \& Critical Care Medicine Journal}

cross-sectional area of the ulnar nerve at the cubital tunnel and an increase in pressure within the cubital tunnel [54]. Elbow flexion of $70^{\circ}$ or more led to a significant increase in the ulnar nerve intraneural pressure [54]. Flexion of the elbow to $135^{\circ}$ resulted in an $18 \%$ elongation of the ulnar nerve [55].The ulnar nerve is surrounded by fat through the cubital tunnel except in the segment adjacent to the medial epicondyle. MRI studies of fresh human cadavers showed that elbow flexion displaced fat posteriorly and relocated the ulnar nerve to a more anterior position against the medial epicondyle in the cubital tunnel [36]. Elevation of the shoulder, flexion of the elbow and dorsiflexion of the wrist stretched the ulnar nerve and led to a marked increase in the intraneural pressure [56].

\section{Identifying High-Risk Patients during Preoperative Anesthetic Evaluation}

As mentioned a variety of risk factors can lead to PPNI. Patient predisposition and precipitating factors can contribute to the injury. The combination of multiple risk factors and precipitating factors in the same patient is concerning and warrants special consideration. For example a patient with a history of hypertension, diabetes, tobacco use, and obesity who presents for a neurosurgical procedure and is expected to have a prolonged hospital stay is likely at increased risk of PPNI. A detailed preoperative history should be obtained from all patients. Patients with risk factors or those who will undergo procedures that are high-risk for PPNI injury deserve a thorough preoperative evaluation. This may include identifying and documenting symptoms suggesting the presence of preexisting peripheral nerve injury and evaluation of the range of motion. The ability of the patient to tolerate the operative position may also be evaluated. Muscular patients should be examined for the maximum comfortable elbow extension. For high riskpatients, PPNI should be included in the perioperative discussion and informed consent process.

\section{The ASA Practice Advisory for the Prevention of Perioperative Peripheral Neuropathies}

Evidence suggests that only a small percentage of perioperative nerve injuries can be definitively associated with positioning and padding [4]. In 1999 the American Society of Anesthesiologists adopted a Practice Advisory for the Prevention of Perioperative Peripheral Neuropathies [6]. At that time only 6 articles out of 509 reviewed had sufficient scientific evidence to link an intervention with an outcome. Thus, the Advisory was written on the basis of expert consultation. Updated Advisories were published in 2011 and 2018 [57,58]. The Advisory is still largely based on expert consensus, although some recommendations are based on level B evidence (noncomparative observational studies or case reports). Advisory Statements regarding the prevention of the upper extremity perioperative peripheral neuropathies are summarized in (Table 2).

\begin{tabular}{|l|}
\hline \multicolumn{1}{|c|}{2018 ASA Advisory for the Prevention of Perioperative Peripheral Neuropathies( ) } \\
\hline Preoperative evaluation \\
\hline It is helpful to ascertain that patients can tolerate the anticipated operative position comfortably \\
\hline Positioning Strategies for the upper extremities to reduce perioperative brachial plexus neuropathy \\
\hline $\begin{array}{l}\text { Arm abduction should be limited to } 90^{\circ} \\
\text { In the prone position, patient may comfortably tolerate arm abduction greater than } 90^{\circ}\end{array}$ \\
\hline Positioning Strategies for the upper extremities to reduce perioperative ulnar nerve neuropathy \\
\hline Supine arms out(on arm board) \\
\hline The arm should be positioned to decrease pressure on the postcondylar(ulnar) groove of the humerus \\
\hline Supination or the neutral forearm positions result in decreased pressure on the ulnar groove \\
\hline Supine arms tucked (at the side) \\
\hline The forearm should be in the neutral position \\
\hline Elbow flexion may increase the risk of ulnar neuropathy(avoid elbow flexion) \\
\hline $\begin{array}{l}\text { There is no consensus on an acceptable degree of flexion during the perioperative period } \\
\text { Positioning Strategies for the upper extremities to reduce perioperative radial nerve neuropathy }\end{array}$ \\
\hline $\begin{array}{l}\text { Prolonged pressure on spiral groove of the humerus should be avoided(may cause radial nerve injury) } \\
\text { Positioning Strategies for the upper extremities to reduce perioperative median nerve neuropathy }\end{array}$ \\
\hline
\end{tabular}




\section{Anaesthesia \& Critical Care Medicine Journal}

\begin{tabular}{l} 
Extension of the elbow beyond the range that is comfortable during the preoperative assessment may stretch the \\
median nerve \\
Periodic assessment of the upper extremity during surgery \\
\hline Periodic perioperative assessment are required to maintain the desired upper extremity position \\
\hline Protective Padding \\
\hline Padding arm boards and the elbow may decrease the risk of PPNI \\
\hline The inappropriate use of padding may increase the risk of PPNI(tight padding) \\
\hline Chest Rolls \\
\hline The use of chest roll in the lateral decubitus position may decrease the risk of PPNI \\
\hline Equipment \\
\hline The use of shoulder braces may increase the risk of PPNI(especially in a steep head-down position) \\
\hline Postoperative Assessment \\
\hline Postoperative assessment of nerve function may lead to early diagnosis of PPNI \\
\hline
\end{tabular}

Table 2: Summary of statement regarding upper extremity perioperative peripheral nerve injury in the ASA practice advisory for the prevention of perioperative peripheral nerve injury.

\section{Positioning Patients for Surgery}

The goal of positioning patients for surgery is to provide optimal operating conditions while maintaining patient safety and minimizing stress on peripheral nerves. Upper extremity positions that lead to stretch or compression of the peripheral nerves should be avoided whenever possible. The head and neck should be maintained in the neutral position while avoiding lateral angulation or rotation. Safety straps should not be tight to avoid direct compression of peripheral nerves and surrounding tissues. The use of shoulder braces should be avoided especially in the steep head-down position. If the use of shoulder braces is deemed necessary, braces should be positioned more laterally against the acromioclavicular joints to reduce direct compression on the brachial plexus. The elbow should not be overextended in a position that would not be tolerated by the patient when awake. Physiologic parameters affecting the perfusion pressure of the peripheral nerve, such as MAP, should be optimized. Based on available knowledge of nerve injury and operative positioning, we advocate the following strategies when positioning the upper extremity for surgery:

\section{The Supine Position}

The supine position is the most common patient position on the operating room table. The patient's arms are positioned either away from the sides on arm boards (supine arms out) or at the sides (supine arms tucked).

In the supine arms out position, there is conflicting literature on a safe level of arm abduction when the arms are placed on arm boards. In spite of this, consulted experts believe that when the arms are abducted on arm boards, abduction should be no more than $90^{\circ}$, and the forearm should be placed in the supine (palms up) or neutral position (palms toward the body). The cubital tunnel of the elbow should be padded to avoid pressure on the ulnar nerve. The wrist should be neutral with respect to the forearm and not extended or flexed. The arm board and padding should be at the same level as the operating room bed and mattress to prevent posterior displacement of the arm. Strategies for positioning the upper extremity in the supine arms out position are summarized in (Table 3).

\begin{tabular}{|c|c|}
\hline \multicolumn{2}{|c|}{ Supine arms out Position } \\
\hline $\begin{array}{c}\text { Head and } \\
\text { neck }\end{array}$ & $\begin{array}{c}\text { Neutral midline. Avoid lateral rotation and } \\
\text { angulation }\end{array}$ \\
\hline \multirow{3}{*}{ Shoulder } & Avoid shoulder braces \\
\cline { 2 - 2 } & Abducted less than $90^{\circ}$ \\
\cline { 2 - 2 } Arm & Avoid posterior shoulder displacement \\
\hline \multirow{2}{*}{ Elbow } & $\begin{array}{c}\text { Avoid full lateral rotation of the arm } \\
\text { Forion beyond the range that is } \\
\text { Forearm }\end{array}$ \\
\cline { 2 - 2 } & Maintain supplyated (preferred) or neutral \\
\hline \multirow{3}{*}{ Wrist } & Neutral in relation to the forearm \\
\cline { 2 - 2 } & Avoid overextension \\
\cline { 2 - 2 } & Apply foam padding \\
\hline
\end{tabular}

Table 3: Upper extremity positioning strategies to minimize compression and stretch of peripheral nerves in the supine arms out position.

In the supine arm tucked position, the arms should be in the neutral position with the palm facing the body. All protruding portions of the arm such as the elbow should 
be protected with padding. Finally, the arm should be protected (with padding or positioning) from all other hard objects. Strategies for positioning the upper extremity in the supine arms tucked position are summarized in (Table 4).

\begin{tabular}{|c|c|}
\hline \multicolumn{2}{|c|}{ Arms tucked positions(supine and prone) } \\
\hline $\begin{array}{c}\text { Head and } \\
\text { neck }\end{array}$ & $\begin{array}{c}\text { Neutral midline. Avoid lateral rotation and } \\
\text { angulation }\end{array}$ \\
\hline Shoulder & Avoid shoulder braces \\
\hline \multirow{2}{*}{ Arm } & $\begin{array}{c}\text { Avoid prolonged shoulder traction (tight } \\
\text { shoulder tape) }\end{array}$ \\
\cline { 2 - 2 } & $\begin{array}{c}\text { Avoid posterior displacement of the shoulder } \\
\text { joint }\end{array}$ \\
\hline Elbow & Avoid lateral rotation of the arm \\
\hline & Apply foam padding \\
\hline Forearm & $\begin{array}{c}\text { Apply foam padding } \\
\text { coid full extension beyond the range that is } \\
\text { comfortable }\end{array}$ \\
\hline Wrist & Neutral \\
\hline & Neutral in relation to the forearm \\
\hline & Avoid overextension \\
\hline
\end{tabular}

Table 4: Upper extremity positioning strategies to minimize compression and stretch of peripheral nerves in the arms tucked positions (supine and prone).

\section{The Prone Position}

Patients are placed in the prone position primarily for spine surgeries and some cranial surgeries. After induction of anesthesia, the patient is placed in the prone position with arms tucked at the sides (prone arms tucked) or placed in front in the "superman" position (prone surrender position). In the prone arms tucked position the safest position for the arms is in the neutral position, palmer surfaces facing the body. Protruding portions of the arms must be padded and the arms generally protected from hard objects. Shoulder traction, usually achieved by taping the shoulders, should be avoided. If shoulder traction is deemed necessary to allow for intraoperative cervical spine imaging studies, it should be minimized. Strategies for positioning the upper extremity in the prone arms tucked position are summarized in Table 4.

In the prone surrender (prone superman) position; the shoulder should be abducted less than $90^{\circ}$, while posterior displacement of the shoulder by gel rolls or supporting structures should be avoided. The arm should be padded to avoid compression of the mid-humeral area by supporting structures such as the Wilson frame. Lateral rotation of the arm should be avoided. The arm board should be at or lower than the level of the operating room table. The elbow should be padded and extreme elbow flexion $\left(>90^{\circ}\right)$ avoided. The forearm should be in neutral or pronated position. The wrist should be in a neutral position in relation to the forearm. Strategies for positioning the upper extremity in the prone surrender position are summarized in (Table 5).

\begin{tabular}{|c|c|}
\hline \multicolumn{2}{|c|}{ Prone "surrender" Position } \\
\hline $\begin{array}{c}\text { Head \& } \\
\text { neck }\end{array}$ & $\begin{array}{c}\text { Neutral midline. Avoid lateral rotation and } \\
\text { angulation }\end{array}$ \\
\hline Shoulder & Avoid shoulder braces \\
\hline & $\begin{array}{c}\text { Abducted less than } 90^{\circ} \\
\text { Avoid posterior displacement of the shoulder } \\
\text { joint }\end{array}$ \\
\hline Arm & $\begin{array}{c}\text { Apply foam padding (between arm and } \\
\text { supporting frame) }\end{array}$ \\
\hline & Avoid lateral rotation of the humerus \\
\hline & Avoid mid-humerus compression \\
\hline Elbow & $\begin{array}{c}\text { Arm board should be at or lower than the level } \\
\text { of the OR table }\end{array}$ \\
\hline & Apply foam padding \\
\hline Forearm & Flexed (avoid extreme flexion $>90^{\circ}$ ) \\
\hline Wrist & Neutral or pronated \\
\hline & Neutral in relation to the forearm \\
\hline
\end{tabular}

Table 5: Upper extremity positioning strategies to minimize compression and stretch of peripheral nerves in the prone surrender position.

\section{The Lateral Decubitus Position}

Patients are placed in the lateral decubitus position for a variety of surgical procedures that include thoracic, spinal and general surgery. When placed in lateral decubitus position the goal is to avoid or minimize compressive forces on the dependent brachial plexus while avoiding stretching of the non-dependent brachial plexus and peripheral nerves. The brachial plexus may be compressed between the humeral head and the thoracic cage [38]. A chest roll should be placed under the dependent chest to reduce compressive forces over the dependent brachial plexus. The chest roll should not be placed under the dependent axilla. A one-liter saline bag wrapped in a towel, or inflatable air bags may be used as chest rolls. Application of an inflatable air bag as a chest 


\section{Anaesthesia \& Critical Care Medicine Journal}

roll has been shown to significantly decrease the pressure under the dependent shoulder from $66 \mathrm{mmHg}$ to 20 mmHg [59]. However, the position of the patient's head and neck should be rechecked and readjusted after application of the chest roll as the average lateral neck angulation has been observed to increase from $14^{\circ}$ to $20^{\circ}$ after chest roll inflation [59]. The arm board supporting the dependent arm and its padding should be at the same level as the operating room table. Shoulder abduction should be maintained less than $90^{\circ}$. The dependent elbow should be extended, while avoiding full or hyperextension. The dependent forearm should be maintained in the supinated position and wrist overextension should be avoided. The arm bar supporting the arm rest for the nondependent arm should be clear of the dependent arm especially the mid-humeral area. The non-dependent arm should be at the level of the non-dependent shoulder and shoulder abduction should be maintained less than $90^{\circ}$. The non-dependent arm should be padded, especially under the elbow, while avoiding extreme elbow flexion $\left(>90^{\circ}\right.$ flexion). The non-dependent forearm is usually in the pronated position. Positioning strategies for the upper extremity in the lateral decubitus position are summarized in (Table 6).

\begin{tabular}{|c|c|}
\hline \multicolumn{2}{|r|}{ Lateral decubitus Position } \\
\hline Head \& neck & Neutral midline. Avoid lateral rotation and angulation \\
\hline & Non-Dependent(ND) Arm \\
\hline Shoulder & Abduction less than $90^{\circ}$ \\
\hline \multirow[t]{4}{*}{ Arm } & Apply foam padding \\
\hline & Arm at the same level of the shoulder joint parallel to the floor \\
\hline & Avoid lateral rotation \\
\hline & The medial edge of the arm rest should not compress the humerus \\
\hline \multirow[t]{3}{*}{ Elbow } & Apply foam padding \\
\hline & Arm at the same level of non-dependent shoulder \\
\hline & Flexed (avoid extreme flexion) \\
\hline \multirow[t]{2}{*}{ Forearm } & Apply foam padding \\
\hline & Pronated \\
\hline \multirow[t]{3}{*}{ Wrist } & Neutral in relation to the forearm \\
\hline & Avoid overextension \\
\hline & Dependent Arm \\
\hline Shoulder & Abduction less than $90^{\circ}$ \\
\hline \multirow[t]{2}{*}{ Chest roll } & Should be placed under the dependent chest (not in the axilla) \\
\hline & Readjust lateral neck angulation after placement of the axillary roll \\
\hline \multirow[t]{2}{*}{ Arm } & Arm board at the same level of the OR bed \\
\hline & Arm bar supporting the ND arm should be clear of the humerus \\
\hline \multirow[t]{2}{*}{ Elbow } & Apply foam padding \\
\hline & Avoid full extension beyond the range that is comfortable \\
\hline \multirow[t]{2}{*}{ Forearm } & Apply foam padding \\
\hline & Maintain supinated (preferred) or neutral \\
\hline \multirow[t]{2}{*}{ Wrist } & Neutral in relation to the forearm \\
\hline & Avoid overextension \\
\hline
\end{tabular}

Table 6: Upper extremity positioning strategies to minimize compression and stretch of peripheral nerves in the lateral decubitus position.

\section{Neuromonitoring}

Multiple authors have reported the successful use of SSEP to monitor the function of peripheral nerves during surgery and to identify and reverse upper extremity neurapraxia under general anesthesia [34,60-75]. Although SSEP monitoring is used primarily to monitor the integrity of the central nervous system during neurosurgical and spinal procedures, it provides the added benefit of monitoring peripheral nerve function. Intraoperative neurapraxia detected by SSEP may indicate impending nerve injury. Modification of the upper extremity position to reduce stretch or compression of peripheral nerve leads to resolution of 


\section{Anaesthesia \& Critical Care Medicine Journal}

neurapraxia and potentially prevents PPNI [34]. Position modification that may lead to reversal of impending nerve injury includes, reducing elbow flexion and extension, abducting the shoulder $<90^{\circ}$, removing restricting arm bands and tape, adding padding to the elbow joint, reducing elbow and wrist extension and returning the arm to the original position, if moving the arm caused the conduction change [34]. Kamel IR, et al. used SSEP to investigate the relationship between intraoperative mean arterial blood pressure (MAP) and upper extremity neurapraxia during spine surgery in the prone surrender position [76].

The authors reported MAP changes as independent predictors for intraoperative upper extremity neurapraxia. MAP below $55 \mathrm{mmHg}$ for 5 or more minutes was significantly associated with higher incidence of intraoperative upper extremity neurapraxia while maintaining the MAP above $80 \mathrm{mmHg}$ for a total duration of 55 or more minutes throughout the case was significantly associated with lower incidence of intraoperative upper extremity nerupraxia [76]. The major limitation of SSEP monitoring is that it is considered a limited resource in most institutions and so is not routinely used to monitor upper extremity nerve function under general anesthesia. Also, only stimulated nerves can be monitored. Thus, it is very unlikely that all peripheral nerves will be stimulated during a surgical case. However, whenever SSEP monitoring is used it should be utilized to identify intraoperative upper extremity neurapraxia.

\section{Postoperative Patient Positioning}

During the postoperative period hospitalized patients are commonly in bed and in the supine position. Their hands and forearms are usually resting on the abdomen or chest while the elbows rest on the bed. Maintenance of this position for a prolonged period may lead to nerve injury especially in high-risk patients. In this position, elbow flexion leads to stretching of the ulnar nerve and the pronated position of the forearm increases pressure on the ulnar nerve at the cubital tunnel. This may partially explain why new onset ulnar nerve injury often manifests days after surgery and why injury to this nerve is also reported in medical patients not undergoing surgical procedures $[14,41,44]$. Patients should be instructed to avoid this position for prolonged periods of time and nurses caring for postoperative patients should be made aware of these potential complications.

\section{Managing PPNI}

Postoperative assessment of upper extremity peripheral nerve function may lead to early diagnosis of PPNI [58]. If a patient develops PPNI, a detailed history and evaluation should be obtained with particular focus on the type (sensory versus motor) and distribution of the deficit. If there is an isolated sensory loss the patient should be followed for 5 days. If the deficit persists for longer than 5 days a neurologist should be consulted. Patients with a motor component to the injury should be seen by a neurologist as soon as possible. Cases with a motor component usually indicate a more severe injury and often a worse prognosis. All management steps should be documented in the patient's medical record.

\section{References}

1. Welch MB, Brummett CM, Welch TD, Tremper KK, Shanks AM, et al. (2009) Perioperative peripheral nerve injuries: a retrospective study of 380,680 cases during a 10-year period at a single institution. Anesthesiology 111(3): 490-497.

2. Cassoria L, Lee JW (2009) Patient positioning in anesthesia. In: Miller RD (Eds) Miller's Anesthesia, ( $7^{\text {th }}$ edn), Elsevier, Philadelphia: 1151-1170.

3. Kroll DA, Caplan RA, Posner K, Ward RJ, Cheney FW (1990) Nerve injury associated with anesthesia. Anesthesiology 73(2): 202-207.

4. Cheney FW, Domino KB, Caplan RA, Posner KL (1999) Nerve injury associated with anesthesia: a closed claims analysis. Anesthesiology 90(4): 1062-1069.

5. Caplan RA (1999) Will we ever understand perioperative neuropathy? A fresh approach offers hope and insight. Anesthesiology 91(2): 335-336.

6. (2000) Practice advisory for the prevention of perioperative peripheral neuropathies: an updated report by the American Society of Anesthesiologists Task Force on prevention of perioperative peripheral neuropathies. Anesthesiology 92(4): 1168-1182.

7. Smith DR, Kobrine AI, Rizzoli HV (1977) Absence of autoregulation in peripheral nerve blood flow. J Neurol Sci 33(3): 347-352.

8. McManis PG, Schmelzer JD, Zollman PJ, Low PA (1997) Blood flow and autoregulation in somatic and 


\section{Anaesthesia \& Critical Care Medicine Journal}

autonomic ganglia. Comparison with sciatic nerve. Brain 120(3): 445-449.

9. Sundqvist T, Oberg PA, Rapoport SI (1985) Blood flow in rat sciatic nerve during hypotension. Experimental Neurol 90(1): 139-148.

10. Low PA, Tuck RR (1984) Effects of changes of blood pressure, respiratory acidosis and hypoxia on blood flow in the sciatic nerve of the rat. J Physiol 347: 513524.

11. Parry GJ, Cornblath DR, Brown MJ (1985) Transient conduction block following acute peripheral nerve ischemia. Muscle Nerve 8(5): 409-412.

12. Myers RR, Yamamoto T, Yaksh TL, Powell HC (1993) The role of focal nerve ischemia and Wallerian degeneration in peripheral nerve injury producing hyperesthesia. Anesthesiology 78(2): 308-316.

13. Bonner SM, Pridie AK (1997) Sciatic nerve palsy following uneventful sciatic nerve block. Anaesthesia 52(12): 1205-1207.

14. Warner MA, Warner ME, Martin JT (1994) Ulnar neuropathy. Incidence, outcome, and risk factors in sedated or anesthetized patients. Anesthesiology 81(6): 1332-1340.

15. Sunderland S (1945) The intraneural topography of the radial, median and ulnar nerves. Brain 68: 243299.

16. Aguayo A, Nair CP, Midgley R (1971) Experimental progressive compression neuropathy in the rabbit. Histologic and electrophysiologic studies. Arch Neurol 24(4): 358-364.

17. Nukada H, McMorran PD (1994) Perivascular demyelination and intramyelinic oedema in reperfusion nerve injury. J Anat 185(2): 259-266.

18. Schmelzer JD, Zochodne DW, Low PA (1989) Ischemic and reperfusion injury of rat peripheral nerve. Proc Natl Acad Sci USA 86(5): 1639-1642.

19. Mäkitie J, Teräväinen $H$ (1977) Peripheral nerve injury and recovery after temporary ischemia. Acta Neuropathol 37(1): 55-63.

20. Nukada H, Powell HC, Myers RR (1992) Perineurial window: demyelination in nonherniated

Kamel I and Huck E. Perioperative Upper Extremity Peripheral Nerve Injury and Patient Positioning: What Anesthesiologists Need to Know. Anaesth Critic Care Med J 2019, 4(3): 000155. endoneurium with reduced nerve blood flow. J Neuropathol Exp Neurol 51(5): 523-530.

21. Swenson JD, Bull DA (1997) Postoperative ulnar neuropathy associated with prolonged ischemia in the upper extremity during coronary artery bypass surgery. Anesth Analg 85(6): 1275-1257.

22. Swenson JD, Hutchinson DT, Bromberg M, Pace NL (1998) Rapid onset of ulnar nerve dysfunction during transient occlusion of the brachial artery. Anesth Analg 87(3): 677-680.

23. Yamada T, Muroga T, Kimura J (1981) Tourniquetinduced ischemia and somatosensory evoked potentials. Neurology 31(12): 1524-1529.

24. Kozu H, Tamura E, Parry GJ (1992) Endoneurial blood supply to peripheral nerves is not uniform. J Neurol Sci 111(2): 204-208.

25. Winfree CJ, Kline DG (2005) Intraoperative positioning nerve injuries. Surg Neurol 63(1): 5-18.

26. Wall EJ, Massie JB, Kwan MK, Rydevik BL, Myers RR, et al. (1992) Experimental stretch neuropathy. Changes in nerve conduction under tension. J Bone Joint Surg Br 74(1): 126-129.

27. Tanoue M, Yamaga M, Ide J, Takagi K (1996) Acute stretching of peripheral nerves inhibits retrograde axonal transport. J Hand Surg Br 21(3): 358-363.

28. Ogata K, Naito M (1986) Blood flow of peripheral nerve effects of dissection, stretching and compression. J Hand Surg Br 11(1): 10-14.

29. Koike $H$ (1987) the disturbance of the fast axonal transport of protein by passive stretching of an axon in Aplysia. J Physiol 390: 489-500.

30. Kwan MK, Wall EJ, Massie J, Garfin SR (1992) Strain, stress and stretch of peripheral nerve. Rabbit experiments in vitro and in vivo. Acta Orthop Scand 63(3): 267-272.

31. Brown R, Pedowitz R, Rydevik B, Woo S, Hargens A, et al. (1993) Effects of acute graded strain on efferent conduction properties in the rabbit tibial nerve. Clin Orthop Relat Res (296): 288-294.

32. Lundborg G (1988) Intraneural microcirculation. Orthop Clin North Am 19(1): 1-12. 


\section{Anaesthesia \& Critical Care Medicine Journal}

33. Rydevik BL, Kwan MK, Myers RR, Brown RA, Triggs $\mathrm{KJ}$, et al. (1990) An in vitro mechanical and histological study of acute stretching on rabbit tibial nerve. J Orthop Res 8(5): 694-701.

34. Kamel IR, Drum ET, Koch SA, Whitten JA, Gaughan JP, et al. (2006) The use of somatosensory evoked potentials to determine the relationship between patient positioning and impending upper extremity nerve injury during spine surgery: a retrospective analysis. Anesth Analg 102(5): 1538-1542.

35. Ogata K, Shimon S, Owen J, Manske PR (1991) Effects of compression and devascularisation on ulnar nerve function. A quantitative study of regional blood flow and nerve conduction in monkeys. J Hand Surg $\mathrm{Br}$ 16(1): 104-108.

36. Patel VV, Heidenreich FP Jr, Bindra RR, Yamaguchi K, Gelberman RH (1998) Morphologic changes in the ulnar nerve at the elbow with flexion and extension: a magnetic resonance imaging study with 3dimensional reconstruction. J Shoulder Elbow Surg 7(4): 368-374.

37. Staff NP, Engelstad J, Klein CJ, Amrami KK, Spinner RJ, et al. (2010) Post-surgical inflammatory neuropathy. Brain 133(10): 2866-2880.

38. Sawyer RJ, Richmond MN, Hickey JD, Jarrratt JA (2000) Peripheral nerve injuries associated with anaesthesia. Anaesthesia 55(10): 980-991.

39. Lee LA, Domino KB (2002) The closed claims project has it influenced the anesthetic practice and outcome? Anesthesiology Clin North Am 20(3): 485501.

40. Chui J, Murkin JM, Posner KL, Domino KB (2018) Perioperative Peripheral Nerve Injury after General Anesthesia: A Qualitative Systematic Review. Anesth Analg 127(1): 134-143.

41. Warner MA, Warner DO, Matsumoto JY, Harper CM, Schroeder DR, et al. (1999) Ulnar neuropathy in surgical patients. Anesthesiology 90(1): 54-59.

42. Sharma AD, Parmley CL, Sreeram G, Grocott HP (2000) Peripheral nerve injuries during cardiac surgery: risk factors, diagnosis, prognosis, and prevention. Anesth Analg 91(6): 1358-1369.
43. Alvine FG, Schurrer ME (1987) Postoperative ulnarnerve palsy. Are there predisposing factors? J Bone Joint Surg Am 69(2): 255-259.

44. Warner MA, Warner DO, Harper CM, Schroeder DR, Maxson PM (2000) Ulnar neuropathy in medical patients. Anesthesiology 92(2): 613.

45. Contreras MG, Warner MA, Charboneau WJ, Cahill DR (1998) Anatomy of the ulnar nerve at the elbow: potential relationship of acute ulnar neuropathy to gender differences. Clin Anat 11(6): 372-378.

46. Warmer ME (2013) Patient positioning and Potential injuries. In: Barash PG, Cullen BF, Stoelting RK (Eds) Clinical anesthesia $7^{\text {th }}$ (Edn.), Lippincot Williams \& Wilkins, Phildelphia: 803-823.

47. Morell RC, Prielipp RC, Harwood TN, James RL, Butterworth JF (2003) Men are more susceptible than women to direct pressure on unmyelinated ulnar nerve fibers. Anesth Analg 97(4): 1183-1188.

48. Cooper DE, Jenkins RS, Bready L, Rockwood CA Jr (1988) The prevention of injuries of the brachial plexus secondary to malposition of the patient during surgery. Clin Orthop Relat Res (228): 33-41.

49. Warner MA (1996) Patient positioning and nerve injury. Anesthesiology clinics 14(3): 561-572.

50. Ngamprasertwong $\mathrm{P}$, Phupong V, Uerpairojkit K (2004) Brachial plexus injury related to improper positioning during general anesthesia. J Anesth 18(2): 132-134.

51. Chowet AL, Lopez JR, Brock-Utne JG, Jaffe RA (2004) Wrist hyperextension leads to median nerve conduction block: implications for intra-arterial catheter placement. Anesthesiology 100(2): 287-291.

52. Prielipp RC, Morell RC, Walker FO, Santos CC, Bennett J, et al. (1999) Ulnar nerve pressure: influence of arm position and relationship to somatosensory evoked potentials. Anesthesiology 91(2): 345-354.

53. Coppieters MW, Van de Velde M, Stappaerts KH (2002) Positioning in anesthesiology: toward a better understanding of stretch-induced perioperative neuropathies. Anesthesiology 97(1): 75-81.

54. Gelberman RH, Yamaguchi K, Hollstien SB, Winn SS, Heidenreich FP Jr, et al. (1998) Changes in interstitial pressure and cross-sectional area of the cubital 


\section{Anaesthesia \& Critical Care Medicine Journal}

tunnel and of the ulnar nerve with flexion of the elbow. An experimental study in human cadavera. J Bone Joint Surg Am 80(4): 492-501.

55. Schuind FA, Goldschmidt D, Bastin C, Burny F (1995) A biomechanical study of the ulnar nerve at the elbow. J Hand Surg Br 20(5): 623-627.

56. Pechan J, Julis I (1975) The pressure measurement in the ulnar nerve. A contribution to the pathophysiology of the cubital tunnel syndrome. J Biomech 8(1): 75-79.

57. American Society of Anesthesiologists Task Force on Prevention of Perioperative Peripheral Neuropathies (2011) Practice advisory for the prevention of perioperative peripheral neuropathies: an updated report by the American Society of Anesthesiologists Task Force on prevention of perioperative peripheral neuropathies. Anesthesiology 114(4): 741-754.

58. American Society of Anesthesiologists Task Force on Prevention of Perioperative Peripheral Neuropathies (2018) Practice advisory for the prevention of perioperative peripheral neuropathies: an updated report by the American Society of Anesthesiologists Task Force on prevention of perioperative peripheral neuropathies. Anesthesiology 128(1): 11-26.

59. Gonzalez Della Valle A, Salonia-Ruzo P, Peterson MG, Salvati EA, Sharrock NE (2001) Inflatable pillows as axillary support devices during surgery performed in the lateral decubitus position under epidural anesthesia. Anesth Analg 93(5): 1338-1343.

60. Mahla ME, Long DM, McKennett J, Green C, McPherson RW (1984) Detection of brachial plexus dysfunction by somatosensory evoked potential monitoring--a report of two cases. Anesthesiology 60(3): 248-252.

61. Pitman MI, Nainzadeh N, Ergas E, Springer S (1988) The use of somatosensory evoked potentials for detection of neuropraxia during shoulder arthroscopy. Arthroscopy 4(4): 250-255.

62. Hickey C, Gugino LD, Aglio LS, Mark JB, Son SL, et al. (1993) Intraoperative somatosensory evoked potential monitoring predicts peripheral nerve injury during cardiac surgery. Anesthesiology 78(1): 29-35.

63. Jellish WS, Martucci J, Blakeman B, Hudson E (1994) Somatosensory evoked potential monitoring of the brachial plexus to predict nerve injury during internal mammary artery harvest: intraoperative comparisons of the Rultract and Pittman sternal retractors. J Cardiothorac Vasc Anesth 8(4): 398-403.

64. Jones SC, Fernau R, WoeltjenBL (2004) Use of somatosensory evoked potentials to detect peripheral ischemia and potential injury resulting from positioning of the surgical patient: case reports and discussion. Spine J 4(3): 360-362.

65. Schwartz DM, Sestokas AK, Hilibrand AS, Vaccaro AR, Bose B, et al. (2006) Neurophysiological identification of position-induced neurologic injury during anterior cervical spine surgery. J Clin Monit Comput 20(6): 437-444.

66. Bethune AJ, Houlden DA, Smith TS, Yee AJ, Midha R, et al. (2007) Generalized peripheral nerve failure during thoracic spine surgery: a case report. J Clin Monit Comput 21(1): 41-47.

67. Anastasian ZH, Ramnath B, Komotar RJ, Bruce JN, Sisti MB, et al. (2009) Evoked potential monitoring identifies possible neurological injury during positioning for craniotomy. Anesth Analg 109(3): 817-821.

68. Chung I, Glow JA, Dimopoulos V, Walid MS, Smisson $\mathrm{HF}$, et al. (2009) Upper-limb somatosensory evoked potential monitoring in lumbosacral spine surgery: a prognostic marker for position-related ulnar nerve injury. Spine J 9(4): 287-295.

69. Schwartz DM, Drummond DS, Hahn M, Ecker ML, Dormans JP (2000) Prevention of positional brachial plexopathy during surgical correction of scoliosis. J Spinal Disord 13(2): 178-182.

70. Jahangiri FR, Holmberg A, Vega-Bermudez F, Arlet V (2011) Preventing position-related brachial plexus injury with intraoperative somatosensory evoked potentials and transcranial electrical motor evoked potentials during anterior cervical spine surgery. Am J Electroneurodiagnostic Technol 51(3): 198-205.

71. Davis SF, Khalek MA, Giles J, Fox C, Lirette L, et al. (2011) Detection and prevention of impending brachial plexus injury secondary to arm positioning using ulnar nerve somatosensory evoked potentials during transaxillary approach for thyroid lobectomy. Am J Electroneurodiagnostic Technol 51(4): 274-279. 


\section{Anaesthesia \& Critical Care Medicine Journal}

72. Jellish WS, Sherazee G, Patel J, Cunanan R, Steele J, et al. (2013) Somatosensory evoked potentials help prevent positioning-related brachial plexus injury during skull base surgery. Otolaryngol Head Neck Surg 149(1): 168-173.

73. O'Brien MF, Lenke LG, Bridwell KH, Padberg A, Stokes M (1994) Evoked potential monitoring of the upper extremities during thoracic and lumbar spinal deformity surgery: a prospective study. J Spinal Disord 7(4): 277-284.

74. Makarov MR, Delgado MR, Birch JG, Samchukov ML (1997) Monitoring peripheral nerve function during external fixation of upper extremities. J Pediatr Orthop 17(5): 663-667.
75. Ohashi N, Furutani K, Ishii H, Baba H (2012) Perioperative brachial plexus injury caused by hyperabduction of the upper extremity in a patient with Ehlers-Danlos syndrome in the prone position. Masui 61(6): 626-628.

76. Kamel I, Zhao H, Koch SA, Brister N, Barnette RE (2016) The use of somatosensory evoked potentials to determine the relationship between intraoperative arterial blood pressure and intraoperative upper extremity position-related neurapraxia in the prone surrender position during spine surgery: A retrospective analysis. Anesth Analg 122(5): 14231433. 